\title{
Ingeniería y Ciencia
}

ISSN:1794-9165 $\mid$ ISSN-e: 2256-4314

ing. cienc., vol. 15, no. 30, pp. 101-116 julio-diciembre. 2019.

http://www.eafit.edu.co/ingciencia

This article is licensed under a Creative Commons Attribution 4.0 by

\section{Medición de la conductividad térmica en materiales aislantes bajo régimen de flujo de calor no estacionario}

\author{
C. M. Daza-Mafioli ${ }^{1}$, E. E. Coral-Escobar ${ }^{2}$ y J. Plaza-Castillo ${ }^{3}$
}

Recepción: 26-08-2019 | Aceptación: 19-11-2019 | En línea: 29-11-2019

PACS:72.80.-r

doi:10.17230/ingciencia.15.30.5

\begin{abstract}
Resumen
Se desarrolló un dispositivo para medir la conductividad térmica en materiales sólidos para aislamiento térmico de uso común en edificaciones, siguiendo un modelo unidimensional de flujo de calor que atraviesa una placa del material a evaluar. Se mide el gradiente de temperatura entre las caras de la placa en función del tiempo, usando un arreglo diferencial de termopares tipo T. Para el control de los mecanismos del instrumento, la adquisición y tratamiento de los datos, se diseñó un circuito con microcontroladores comerciales. En ensayos previos con algunos materiales, se obtuvieron valores la conductividad térmica similares a los reportados en la literatura utilizando un ajuste lineal con valores de $\mathrm{R}$ entre $0.90 \mathrm{y}$ 0.98. El dispositivo obtenido respresenta un instrumento útil para la medición de la conductividad térmica, destacando entre sus ventajas: la fácil
\end{abstract}

\footnotetext{
${ }^{1}$ Universidad del Atlántico, crisdazamafioli@hotmail.com, ORCID:0000-0002-06553285, Barranquilla, Colombia.

${ }^{2}$ Universidad del Atlántico, eulercoral@mail.uniatlantico.edu.co, ORCID:0000-00020329-1013, Barranquilla, Colombia.

${ }^{3}$ Universidad del Atlántico, jairoplaza@mail.uniatlantico.edu.co, ORCID:0000-00026135-7578, Barranquilla, Colombia.
} 
Medición de la conductividad térmica en materiales aislantes bajo régimen de flujo de calor no estacionario

construcción, el tamaño de las muestras a evaluar y el método de medida, comparado con los métodos tradicionales para medir este parámetro.

Palabras clave: Conductividad térmica; flujo de calor; aislantes térmicos; incertidumbre; consumo energético; construcción de edificaciones.

\title{
Thermal Conductivity Measurement of Insulating Materials Under Non-stationary Heat Flow
}

\begin{abstract}
A device to measure thermal conductivity in thermal insulating solid materials commonly used in buildings was developed following a one-dimensional model of heat flow through a plate of the material to be evaluated. The temperature gradient between the plate faces was measured as a function of time by means of a set of type T thermocouples. A circuit with commercial microcontrollers was designed to control the instrument's mechanisms, the acquisition and the treatment of data. In preliminary tests with some materials, thermal conductivity values similar to those reported in the literature were obtained by using a linear adjustment with $\mathrm{R}$ values between 0.90 and 0.98 . This device turns out to be a good instrument for measuring thermal conductivity because it has several advantages, such as: easy implementation, sample size, measurement method; compared to those using traditional methods.
\end{abstract}

Keywords: Thermal conductivity; heat flow; thermal insulator; uncertainty; energy consumption; building construction.

\section{Introducción}

Las tendencias actuales en el diseño y construcción de edificaciones, consideran el consumo energético empleado para generar ambientes más confortables en climas calientes y fríos. Para disminuir este consumo energético, es necesario emplear aislantes térmicos [1].

Para evaluar el coeficiente de conductividad térmica (CT) de materiales que se usan en construcciones a nivel local, se fabricó un dispositivo a partir de un equipo sencillo de uso didáctico [2], pero con su mecanismo y toma de datos controlados por un microcontrolador. Un primer diseño se desarrolló teniendo en cuenta que las dimensiones de todas las partes cupieran dentro de una campana de vidrio que permitiera realizar mediciones de la conductividad térmica tanto al aire como en bajo vacío [3], 4]. 
En un segundo proyecto, se modificó el diseño del instrumento y el circuito de control electrónico, automatizando totalmente el proceso de medida con el fin de garantizar la reproducibilidad del experimento. Además se implementó un algoritmo matemático para el tratamiento de los datos experimentales, mostrando en una pantalla el valor del parámetro de interés y la incertidumbre de la medida.

En la literatura se encuentran trabajos de medición de la conductividad térmica de materiales aislantes entre los que se distinguen tres métodos de acuerdo a la dependencia temporal de la temperatura durante la medición: El primero es el de estado estacionario, en el cual se crea un gradiente de temperatura constante entre las dos caras de la muestra. Un segundo método es el de pulso periódico de calor y el tercero es el transitorio o de estado no estacionario, en el cual el gradiente es dependiente del tiempo y de la posición y de acuerdo con W. P. Adamczyk [5], este método es más rápido y se adquieren más datos que en el estado estacionario. En este sentido, Ricciu et al. [6] muestran una revisión de los métodos usados para caracterizar térmicamente materiales de construcción.

En muchos trabajos en los que se mide la conductividad térmica de materiales aislantes, el método más común es el de placa caliente guardada, establecido por la norma ASTM C 1363 [7, [8]. En estos se establece un gradiente de temperatura constante por lo cual el flujo de calor a través de la muestra se mantiene en régimen estacionario. Por ejemplo, A. Hadded et al. [9]. determinan la conductividad térmica y la difusividad de láminas aislantes fabricadas con residuos de textiles. Las láminas tienen $2 \mathrm{~cm}$ de espesor y deben esperar 3 y $6 \mathrm{~h}$ para lograr un régimen estacionario. Sus resultados muestran una baja conductividad por lo cual los materiales producidos se pueden considerar como aislantes térmicos. Santa et al. [10] usan un dispositivo para medir la conductividad térmica en materiales aislantes por el método de placa guardada. Gomes et al. [11] usan dos métodos para comparar sus resultados: régimen estacionario y transitorio, obteniendo diferentes resultados con ambos métodos. Buratti et al. [7] desarrollaron un equipo consistente en una caja con un panel caliente y otro frío, para caracterizar materiales compuestos obteniendo valores del $\mathrm{CT}$ en rangos adecuados para considerarlos como aislantes térmicos prometedores en remodelación de edificios. Por su parte Jannot et al. [12] presentan un método de pulso de calor para medir el CT en materiales aislantes de baja densi- 
dad, argumentando ser mejor que el método de régimen no estacionario para estos materiales. Su método consiste en introducir la muestra entre dos placas conductoras y miden las temperaturas de cada placa después de aplicar un pulso de calor en una de ellas, obteniendo así buena precisión en las medidas. La referencia [13] presenta una revisión de los materiales aislantes térmicos usados en edificaciones con sus valores característicos del CT, la cual se tomó como fuente de información para comparar los resultados obtenidos. Se resalta el trabajo de E. Yamasue et al. [14] ya que ellos implementan el método de flujo de calor no estacionario para medir el CT de silicio y germanio mediante análisis de la relación lineal entre el gradiente de temperatura y el logaritmo del tiempo, este es el mismo concepto utilizado en el presente trabajo para obtener el valor del CT.

En este trabajo se utilizó el método de placa caliente y placa fría entre las que se coloca el material a evaluar considerando un flujo de calor unidireccional con gradiente de temperatura variable en el tiempo, por lo que las mediciones se realizaron en estado no estacionario permitiendo una fácil implementación del sistema y de la adquisición de los datos. El modelo experimental seguido, es el mismo expuesto en Meiners [2], con una variante en la medición de la temperatura como función de la diferencia de potencial y no de la corriente eléctrica arrojada por el termopar diferencial como función del tiempo.

\section{Modelo teórico}

Se considera un flujo de calor unidimensional a través de un material isotrópico, en forma de placa de espesor $L$ y sección transversal $A$ cuyas caras están a las temperaturas $T_{1}$ y $T_{2}\left(T_{1}>T_{2}\right)$ dado por

$$
\frac{d Q}{d t}=-k A \frac{T_{2}-T_{1}}{L}
$$

en donde $\mathrm{k}$ es el coeficiente de conductividad térmica, característica de cada material e indica la cantidad de calor que pasa a través de la muestra por unidad de temperatura, de tiempo y de longitud. Esta ecuación es conocidad como la ley de Fourier [15]. El calor que fluye a través de la placa es recolectado por un bloque de cobre y es proporcional al cambio de 
su temperatura, a su masa m y a su calor específico $c_{p}$. La razón a la que el cuerpo acumula el calor está dada por

$$
\frac{d Q}{d t}=m c_{p} \frac{d T}{d t}
$$

De acuerdo con estas dos ecuaciones y considerando que no hay pérdidas de calor en el sistema (ya que en proporción estas son del $0.01 \%$ del calor acumulado por el calorímetro en cada instante de tiempo), se concluye que el calor que pasa a través de la placa en la unidad de tiempo es igual al calor que acumula el bloque de cobre cada segundo.

La diferencia de temperatura entre las caras de la placa es proporcional a la diferencia de potencial generada por los termopares en contacto con el calentador y el bloque de cobre por efecto Seebeck [16] y se expresa como $V=C\left(T_{2}-T_{1}\right)$, donde $C$ es una constante de proporcionalidad. Como la temperatura $T_{2}$ varía con el tiempo, las variaciones instantáneas del potencial se obtienen según la ecuación 3.

$$
\frac{d V}{d t}=-C \frac{d T}{d t}
$$

Relacionando las ecuaciones 1, 2, y 3 se obtiene una ecuación diferencial sencilla que al integrarse resulta una ecuación lineal en términos de logaritmos (ecuación 4) cuya pendiente depende de la masa y el calor específico del bloque de cobre, de la conductividad térmica $\mathrm{k}$, el espesor L y el área de contacto A del material a evaluar.

$$
\log V=\log V_{0}-\frac{k A}{m c_{p} L} t
$$

Obteniendo el valor de la pendiente $M$ a partir de esta ecuación, se obtiene el valor de la conductividad térmica normalizada al espesor de cada muestra, ecuación 5 .

$$
\frac{k}{L}=\frac{M c_{p} m}{A}
$$

El valor de esta pendiente se obtiene experimentalmente, registrando el voltaje de los termopares como una función del tiempo y aplicando el método de mínimos cuadrados como se expone en la referencia [17]. 
La incertidumbre en la medición se calcula mediante la ley de propagación de incertidumbres, ecuación 6 [18],[19],

$$
u_{\frac{k}{L}}=\sqrt{\left(\frac{\partial f}{\partial A} u_{A}\right)^{2}+\left(\frac{\partial f}{\partial m} u_{m}\right)^{2}+\left(\frac{\partial f}{\partial b} u_{M}\right)^{2}}
$$

y dependerá de la incertidumbre de cada variable independiente: área de transferencia, masa del calorímetro y de la pendiente calculada con el método de míninos cuadrados. Al realizar un desarrollo algebraico de esta ecuacion se obtiene una expresión (ecuación 7) que solo depende de la pendiente $\mathrm{M}$ y su incertidumbre $u_{M}$ ya que las demás incertidumbres son constantes, por lo que a mayor desviación en los datos y mayor pendiente (mayor conductividad de la muestra) mayor será la incertidumbre de la medida.

$$
u_{\frac{k}{L}}=385 \sqrt{9.198(M)^{2}+30115.391\left(u_{M}\right)^{2}}
$$

\section{Diseño del instrumento}

El sistema está constituido por un calentador y un bloque de cobre entre los cuales se coloca un disco delgado de la muestra a evaluar con un área mayor a la del bloque. Se utilizan seis termopares diferenciales tipo T (cobreconstantan) soldando tres de ellos en un punto interior del bloque de cobre (calorímetro) y los otros tres en puntos diferentes del calentador, con los cuales se determina la diferencia de temperatura entre las caras del material de prueba como se observa en la Figura 1. La temperatura medida en $m V$ de cada termopar es promediada y este valor se almacena y se procesa con un microcontrolador mediante un algoritmo de cálculo. 


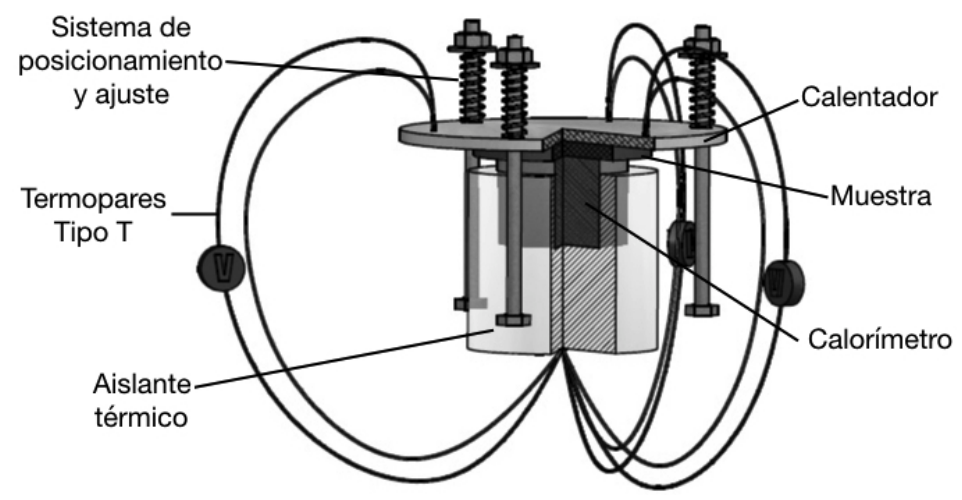

Figura 1: Esquema de la sección principal del medidor de conductividad.

\subsection{Sistema de calefacción}

El calentador se implementó con resistencias cerámicas adheridas a la cara superior de una placa de aluminio sobre la que se soldaron los tres termopares. En el centro de esta cara del calentador se adhiere un sensor LM35 para realizar el control de la temperatura (Figura 2).

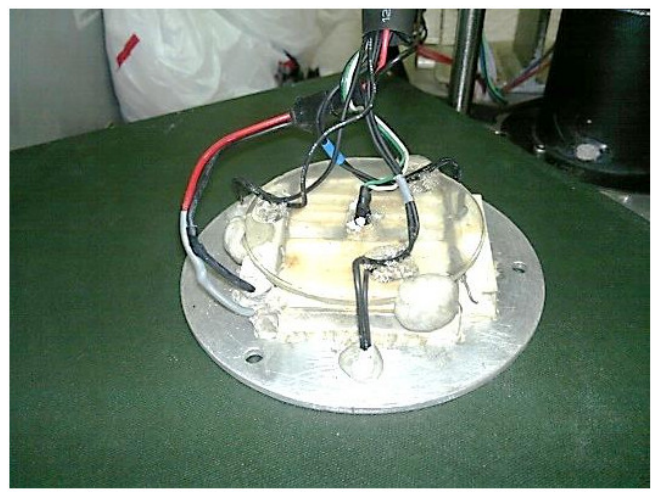

Figura 2: Calentador con termopares y sensor LM35.

ing.cienc., vol. 15, no. 30, pp. 101 116 julio-diciembre. 2019. 


\subsection{Operación del mecanismo}

Teniendo en cuenta que el dispositivo se proyectó para hacer medidas en una campana de vacío, se diseñó un mecanismo de piñón-cremallera para subir y bajar el calentador el cual se encuentra sujeto mediante tres resortes en un cilindro con material aislante(Figura 3). Los resortes permiten que la muestra haga buen contacto con las superficies del calentador y el bloque de cobre y se adapte a los espesores de la placa a evaluar ya que solo pueden estar entre 1 y $5 \mathrm{~mm}$ debido al limitante de la geometría de equipo y la consideración de transferencia de calor unidireccional .

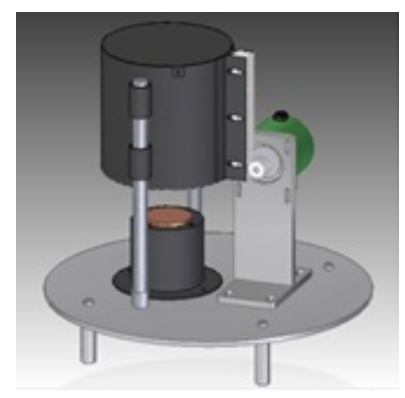

(a) Calentador arriba

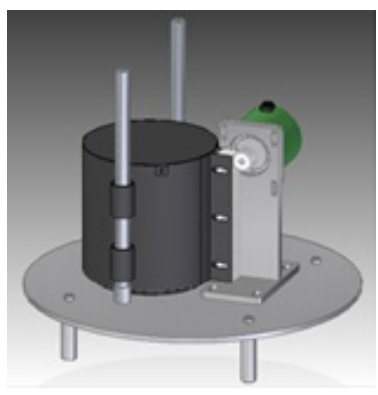

(b) Calentador abajo

Figura 3: Diseño final: (a)calentador elevado y (b) En posición de operación.

\subsection{Sistema de control y medición}

Una vez evaluado el modelo matemático y procedimiento para operar el sistema de medición, el siguiente paso fue elaborar un algoritmo para el funcionamiento del instrumento. La rutina inicia estableciendo las condiciones iniciales de posicionamiento y estabilidad de la temperatura del calentador a la cual se realizará la medición, luego baja el calentador y lo pone en contacto con la muestra y se espera unos segundos para que se establezca el flujo de calor a través de la muestra. Pasado este tiempo, el programa entra en un bucle en donde el microcontrolador principal (PIC 18f252) toma la lectura de la diferencia de $\operatorname{potencial}(V)$ de los tres termopares de 
manera independiente y registra el promedio del valor de las tres lecturas. Este bucle tiene un periodo de 1 segundo. Una vez dentro del bucle, se evalúa la variable dependiente $\ln V$ y se registra cada segundo en función del tiempo $t$ medido mediante los ciclos del oscilador del microcontrolador.

El comparador análogo-digital de 10 bit del microcontrolador PIC18f252 a un voltaje de referencia de $5 \mathrm{~V}$ proporciona una resolución aproximada de $0.005 \mathrm{~V}$ y al tener una etapa de amplificación X1000, se obtiene una lectura de los termopares con una sensibilidad de $0.005 \mathrm{mV}$. Teniendo en cuenta esta sensibilidad, el instrumento podrá realizar mediciones de un material con coeficiente de conductividad térmica inferiores a $3.2 \mathrm{~W} / \mathrm{mK}$ tomando por lo menos 100 datos. Sin embargo, no es recomendado evaluar materiales con conductividad inferior a $0.03 \mathrm{~W} / \mathrm{mK}$, ya que el microcontrolador requeriría de tiempos muy largos para percibir las variaciones en el voltaje de los termopares y en estas condiciones, otros factores como son la estabilidad de las fuentes de voltaje, variación en la carga de instrumento y variación en la temperatura del calentador debido al sistema de control de temperatura, que normalmente no afectan la medida de voltaje, si podrían tener mayor influencia generando variación en la medida.

La pendiente $M$ y la incertidumbre $u_{M}$ son calcularon con el método de mínimos cuadrados, programado en la rutina del microcontrolador y con estos datos se obtiene el valor de la conductividad térmica normalizada al espesor $\frac{k}{L}$, la incertidumbre $u_{\frac{k}{L}}$ y por último el factor de correlación $R$. Al multiplicar los valores normalizados por el espesor de la muestra se determina la conductividad térmica del material en unidades de $W / m K$.

\subsection{Automatización del proceso}

El medidor de conductividad térmica es controlado por un circuito electrónico basado en microcontroladores PIC18f252, PIC12f675 y PIC12f629 todos programados en lenguaje $\mathrm{C}^{++}$, los cuales realizan las tareas de control de los mecanismos del calentador, de la adquisición y almacenamiento de datos y el tratamiento matemático para obtener el parámetro de interés. Cada uno de estos microcontroladores fue programado para ejecutar funciones específicas consiguiendo con esto que trabajen en una configuración maestro-esclavo, cada etapa del proceso en el instrumento es comandada

ing.cienc., vol. 15, no. 30, pp. 101116 julio-diciembre. 2019. 
Medición de la conductividad térmica en materiales aislantes bajo régimen de flujo de calor no estacionario

por el microcontrolador principal (Maestro) PIC18f252; de esta manera se consigue automatizar el instrumento. En la Figura 4 se muestra el esquema de bloques del circuito de control y medidas.

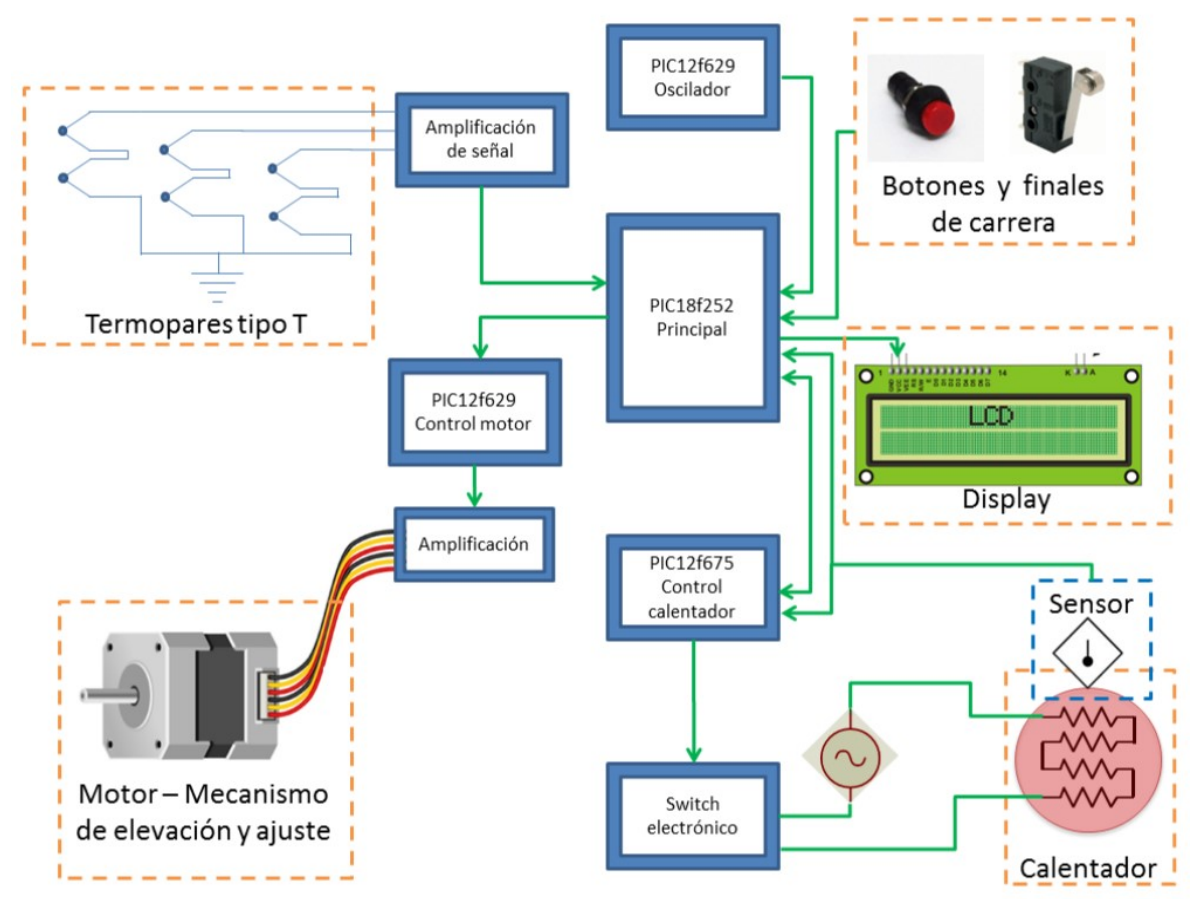

Figura 4: Diagrama de bloques del dispositivo.

\section{Resultados y discusión}

\subsection{Verificación de la medición}

La estabilidad de la temperatura del calentador con control de tipo "on off" se evaluó en múltiples pruebas, concluyendo que el rango de trabajo para el calentador es de 70 a $120^{\circ} \mathrm{C}$ con una variación de $\pm 1^{\circ} \mathrm{C}$.

La primera prueba de medición con nuestro protoptipo se hizo con una lámina de madera de $4.03 \mathrm{~mm}$ de espesor. Los valores de voltaje arrojados 
por los termopares fueron registrados en función del tiempo y con ellos se realizó una gráfica en Excel, como se muestra en la Figura 5. Estos datos fueron ajustados a una función exponencial con un factor de correlación de 0.96 .

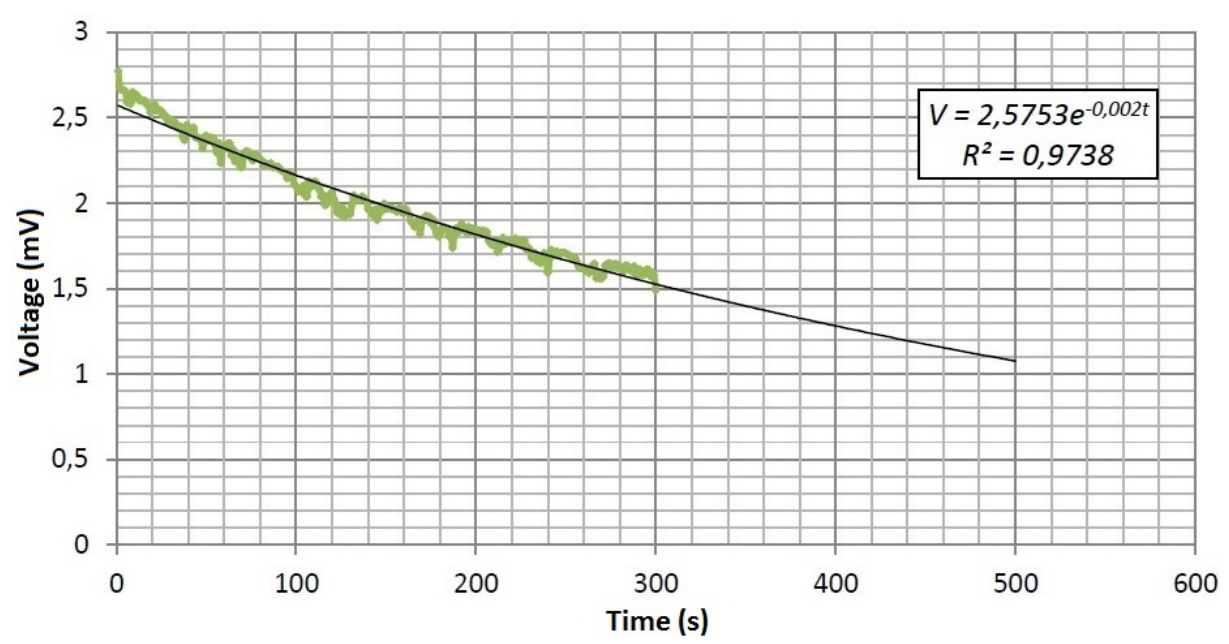

Figura 5: Ajuste exponencial de los datos de voltaje vs. tiempo en una medición de prueba con madera.

Un gráfico del logaritmo del voltaje respecto al tiempo se muestra en la Figura 6 en la que se hizo un ajuste lineal con alguna dispersión pero con un factor de relación aceptable. De la pendiente de esta recta y mediante la ecuación 5, se calculó el valor del coeficiente de conductividad térmica teniendo en cuenta los valores del espesor de la muestra, y los parámetros del bloque de cobre ( área de trasferencia, masa y calor específico). El valor promedio obtenido para la conductividad térmica de la madera fue de $(0.147 \pm 0.004) \frac{W}{m K}$, siendo este un valor muy cercano al reportado en diferentes tablas [20, [21] Cabe resaltar que las gráficas de este experimento fueron una prueba del método de medición ya que el dispositivo no genera una tabla de datos del V vs t, porque el microcontrolador se programó para realizar todo el proceso de adquisición y procesamiento de datos $\mathrm{y}$ presentar el cálculo resultante en una pantalla LCD. 
Medición de la conductividad térmica en materiales aislantes bajo régimen de flujo de calor no estacionario

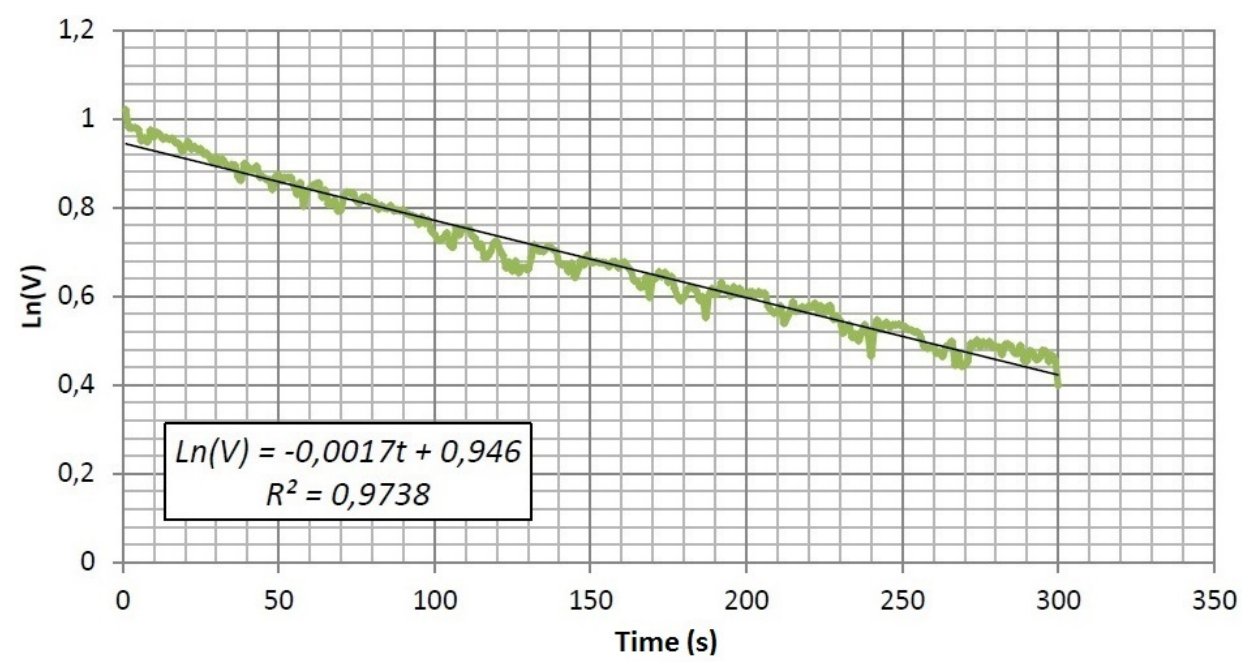

Figura 6: Ajuste lineal para $\operatorname{lnV}$ vs. t.

\subsection{Pruebas con distintos materiales}

Se realizaron pruebas con otros materiales tales como corcho, vidrio y concreto con distintos espesores para verificar el correcto funcionamiento del instrumento, encontrar las limitaciones del proceso de medición y determinar factores que afectan su desempeño. La Tabla 1 muestran los resultados obtenidos con estos materiales. En la columna 1 aparece la temperatura de operación de las mediciones para cada material. Esto fue necesario hacerlo debido a las características de cada material para controlar el tiempo de muestreo. Para modificar la temperatura fue necesario reprogramar el microcontrolador. El número de datos también se tuvo en cuenta. En el caso de una lámina de corcho de $1.72 \mathrm{~mm}$ de espesor, se relizaron mediciones con 600 y 900 datos pero esto no afectó el valor obtenido.

Para madera se usaron 300 y 900 datos, obteniéndose una pequeña variación. Con el vidrio se consiguió evaluar dos espesores de 2.08 y $4.80 \mathrm{~mm}$ se usaron 100 datos y una temperatura de $100^{\circ} \mathrm{C}$, obteniéndose valores de $\mathrm{k}$ muy similares. Para el concreto también fue necesario usar una temperatura de $100^{\circ} \mathrm{C}$ y un muestreo de 300 datos obteniéndose valores de $\mathrm{k}$ en el rango reportado en la literatura al igual que para los demás materiales [1],[13]. 
Tabla 1: Conductividad térmica de alguno materiales

\begin{tabular}{lcccc}
\hline $\left.\begin{array}{l}\text { Material } \\
(\text { Temp }\end{array}{ }^{\circ} \mathrm{C}\right)$ & $\mathrm{R}$ & $\begin{array}{c}\mathrm{k}_{\text {exp }} \\
\left(\frac{W}{m K}\right)\end{array}$ & $\begin{array}{c}u_{k} \\
\left(\frac{W}{m K}\right)\end{array}$ & $\begin{array}{c}\mathrm{k}_{\text {repotado }} \\
\left(\frac{W}{m K}\right)\end{array}$ \\
\hline \hline Corcho & 0.91 & 0.033 & 0.001 & 0.036 \\
$(80)$ & 0.90 & 0.034 & 0.002 & 0.036 \\
\hline Madera & 0.93 & 0.036 & 0.006 & 0.036 \\
$(80)$ & 0.96 & 0.145 & 0.004 & 0.14 \\
\hline Vidrio & 0.97 & 0.917 & 0.039 & $0.6-1.0$ \\
$(100)$ & 0.95 & 0.931 & 0.039 & $0.6-1.0$ \\
\hline Concreto & 0.97 & 0.260 & 0.007 & $0.16-1.4$ \\
$(100)$ & 0.98 & 0.247 & 0.007 & $0.16-1.4$ \\
\hline
\end{tabular}

\section{Conclusiones}

El prototipo obtenido muestra buenos resultados en la medición de conductividad térmica en el rango de 0.03 a $3.2 \mathrm{~W} / \mathrm{mK}$, puesto que en las pruebas, la diferencia de las medias obtenidas respecto a las reportadas en la literatura y obtenidas por otros métodos, tiene un error entre el 3 al $8 \%$. Las mediciones realizadas en madera, corcho, vidrio y concreto se encuentran dentro de los rangos reportados por lo cual se puede considerar que cualquier material aislante térmico sólido para construcción se puede caracterizar en el dispositivo, teniendo en cuenta factores que disminuyen la incertidumbre de la medida; como disminuir el espesor de la muestra en materiales con muy baja conductividad térmica para aumentar la variación de temperatura.

La medición de la conductividad térmica de materiales termoplásticos, como el caso del PVC y el acrílico, es posible siempre y cuando la temperatura de operación del instrumento no produzca cambios en las propiedades físicas de estos materiales. Se encontró que para mejorar la medición, la temperatura y el tiempo de muestreo se deben ajustar para cada material.

Entre las mejoras proyectadas para el instrumento, se incluirá un menú para que el usuario seleccione la temperatura del calentador y tiempo de 
muestreo y se considera implementar un control de temperatura de tipo Proporcional Integral Derivativo (PID), que permita hacer pruebas en materiales que requieran temperaturas más bajas y tener mejor estabilidad de la temperatura.

Se puede afirmar que este sistema de medida es comparable con los indicados en las referencias consultadas ya que se observa que con cada diseño experimental se obtienen valores de coeficientes de conductividad térmica muy próximos entre si. La diferencia se encuentra en que la construcción del dispositivo, la toma de datos y el tratamiento de los datos es de fácil implementación, comparado con los dispositivos de medición más comunes. $\mathrm{Y}$ en cuanto al método de medida, se puede decir que es comparable con el citado en la referencia [14].

El sistema desarrollado tiene gran aplicación para fines didácticos y a nivel industrial, por lo que en un futuro estará ensayándose con materiales cuya conductividad térmica sea conocida y certificada y que puedan usarse como patrones de calibración.

\section{Agradecimientos}

Los autores agradecen a la Universidad del Atlántico por el soporte económico para este proyecto en el marco de la convocatoria Pensar el Caribe.

\section{Referencias}

[1] L. Aditya, T. Mahlia, B. Rismanchi, H. Ng, M. Hasan, H. Metselaar, O. Muraza, and H. Aditiya, "A review on insulation materials for energy conservation in buildings," Renewable and Sustainable Energy Reviews, vol. 73, pp. 1352 - 1365, 2017. [Online]. Available: https: //doi.org/10.1016/j.rser.2017.02.034 102,112

[2] H. F. Meiners, W. Eppenstein, and K. H. Moore, Experimentos de física. Limusa, 1980. 102, 104

[3] I. Morales and E. Carrillo, Diseño y Construcción de un Conductímetro con Control Basado en Tecnología PIC Para la Medición de Conductividad Térmica en Materiales Sólidos. Universidad del Atlántico, 2005. 102 
[4] E. Coral, I. Morales, E. Carrillo, and J. Plaza, "Diseño del sistema de medición de conductividad térmica de materiales para construcción," Revista colombiana de física, vol. 38, no. 3, 2006. [Online]. Available: https://www.cenam.mx/sm2010/info/carteles/sm2010-c32.pdf 102

[5] W. P. Adamczyk, S. Pawlak, and Z. Ostrowski, "Determination of thermal conductivity of cfrp composite materials using unconventional laser flash technique," Measurement, vol. 124, pp. 147 - 155, 2018. [Online]. Available: https://doi.org/10.1016/j.measurement.2018.04.022 103

[6] R. Ricciu, L. A. Besalduch, A. Galatioto, and G. Ciulla, "Thermal characterization of insulating materials," Renewable and Sustainable Energy Reviews, vol. 82, pp. 1765 - 1773, 2018. [Online]. Available: https://doi.org/10.1016/j.rser.2017.06.057 103

[7] C. Buratti, E. Belloni, L. Lunghi, A. Borri, G. Castori, and M. Corradi, "Mechanical characterization and thermal conductivity measurements using of a new 'small hot-box' apparatus: innovative insulating reinforced coatings analysis," Journal of Building Engineering, vol. 7, pp. 63 - 70, 2016. [Online]. Available: https://doi.org/10.1016/j.jobe.2016.05.005 103

[8] X. M. Arce, F. E. Navarrete, and E. I. M. Haro, "Diseño de una caja caliente bajo la norma astm c 1363," Revista Ciencia UNEMI, vol. 9, no. 21, pp. 83-96, 2016. 103

[9] A. Hadded, S. Benltoufa, F. Fayala, and A. Jemni, "Thermo physical characterisation of recycled textile materials used for building insulating," Journal of Building Engineering, vol. 5, pp. 34 - 40, 2016. [Online]. Available: https://doi.org/10.1016/j.jobe.2015.10.007 103

[10] G. D. Santa, F. Peron, A. Galgaro, M. Cultrera, D. Bertermann, J. Mueller, and A. Bernardi, "Laboratory measurements of gravel thermal conductivity: An update methodological approach," Energy Procedia, vol. 125, pp. 671 677, 2017. [Online]. Available: https://doi.org/10.1016/j.egypro.2017.08.287 103

[11] M. G. Gomes, I. Flores-Colen, F. da Silva, and M. Pedroso, "Thermal conductivity measurement of thermal insulating mortars with eps and silica aerogel by steady-state and transient methods," Construction and Building Materials, vol. 172, pp. 696 - 705, 2018. [Online]. Available: https://doi.org/10.1016/j.conbuildmat.2018.03.162 103

[12] Y. Jannot, A. Degiovanni, and G. Payet, "Thermal conductivity measurement of insulating materials with a three layers device," International Journal of Heat and Mass Transfer, vol. 52, no. 5, pp. 1105 - 1111, 2009. [Online]. Available: https://doi.org/10.1016/j.ijheatmasstransfer.2008.09.017 103 
Medición de la conductividad térmica en materiales aislantes bajo régimen de flujo de calor no estacionario

[13] S. Schiavoni, F. D'Alessandro, F. Bianchi, and F. Asdrubali, "Insulation materials for the building sector: A review and comparative analysis," Renewable and Sustainable Energy Reviews, vol. 62, pp. 988 - 1011, 2016. [Online]. Available: https://doi.org/10.1016/j.rser.2016.05.045 104, 112

[14] E. Yamasue, M. Susa, H. Fukuyama, and K. Nagata, "Thermal conductivities of silicon and germanium in solid and liquid states measured by nonstationary hot wire method with silica coated probe," Journal of Crystal Growth, vol. 234, no. 1, pp. 121 - 131, 2002. [Online]. Available: https://doi.org/10.1016/S0022-0248(01)01673-6 104,114

[15] Y. A. Çengel and A. J. Ghajar, Transferencia de calor y masa. Fundamentos y aplicaciones, 4th ed. McGraw Hill, 2011. 104

[16] F. W. Sears and Zemansky, Física general, versión española de Albino Yusta Almarza. Aguilar, 1975. 105

[17] D. C. Baird, Experimentación: una introducción a la teoría de mediciones y al diseño de experimentos. Prentice-Hall Hispanoamericana Mexico. DF, 1991, no. QC39 B3418 1991. 105

[18] W. A. Schmid, R. Lazos et al., "Guía para estimar la incertidumbre de la medición," Centro nacional de Metrología (Abril 2004), 2000. 106

[19] M. M. P. Hernández, "Estimación de incertidumbres. guía gum," Revista Española de Metrología, vol. 1, no. 3, pp. 113-130, 2012. [Online]. Available: https://www.uv.es/meliajl/Docencia/WebComplementarios/ GuiaGUM_e_medida.pdf 106

[20] T. G. Ríos Soto, "Concepción y construcción de un dispositivo para medir la conductividad térmica de materiales para edificaciones," Master's thesis, Universidad de Sonora .División de ingeniería, 1996. [Online]. Available: http://www.bidi.uson.mx/TesisIndice.aspx?tesis=7609 111

[21] L. M. Vélez Moreno, Materiales industriales: teoría y aplicaciones. Medellín: Instituto Tecnológico Metropolitano, 2008. 111 